\title{
Effect of Hand Dominance on Functional Status and Recovery of Hand in Stroke Patients
}

\author{
Pınar Muge Sarikaya ${ }^{1}$, Nurgul Arinci Incel ${ }^{1, ~ *, ~ A r d a ~ Y i l m a z ~}{ }^{2}$, Ozlem Bolgen Cimen ${ }^{1}$, Gunsah Sahin ${ }^{1}$ \\ ${ }^{1}$ Department of PM \& R, Mersin University, School of Medicine, Mersin, Turkey \\ ${ }^{2}$ Department of Neurology, Mersin University, School of Medicine, Mersin, Turkey
}

Email address:

nincel@hotmail.com (N. A. Incel)

${ }^{*}$ Corresponding author

\section{To cite this article:}

Pınar Müge Sarikaya, Nurgül Arinci Incel, Arda Yilmaz, Özlem Bolgen Cimen, Gunsah Sahin. Effect of Hand Dominance on Functional Status and Recovery of Hand in Stroke Patients. Science Journal of Clinical Medicine. Vol. 6, No. 3, 2017, pp. 39-45.

doi: $10.11648 /$ j.sjcm.20170603.12

Received: June 6, 2017; Accepted: June 21, 2017; Published: July 24, 2017

\begin{abstract}
Stroke is characterized by hemiplegia, including motor deficits and various neurological manifestations mainly in contralateral half of the body lasting more than 24 hours with a presumed vascular cause. The goal of stroke rehabilitation is to make people independent and this is possible with appropriate functioning of the affected hand and upper extremity. Stroke patients have to depend on the nondominant hand when the effected hand is the dominant side. However this disadvantage might end up in better results with more effort for recovery of the dominant hand. In this study, we aimed to understand the effect of hand dominance on both functional loss and regain after stroke. 18 patients with right or left hemiplegia participated in the study. Patients were grouped as dominant side and nondominant side hemiplegic (groups D and ND). Patients were evaluated in the $1^{\text {st }}$ week, $1^{\text {st }}$ month and $3^{\text {rd }}$ month. Brunnstrom stages, Motricity index, MAS and FIM, NHPT and hand grip strength were recorded for every patient in every visit. None of the parameters showed significant difference between two groups. Only NHPT used to assess fine manual dexterity revealed a significant difference in the final analysis. Correlation analysis displayed a positive correlation between hand grip strength with FIM and pinch grip strength with NHPT. We concluded that there was no significant difference in functional improvement between dominant and non-dominant side hemiplegic groups during the first three months after stroke. However longer follow ups and larger patient groups are needed to clarify the effect of hand dominance on long term functional status.
\end{abstract}

Keywords: Stroke, Hemiplegia, Dominant Hand, Grip Strength, NHPT, Dexterity, Hand Dominance

\section{Introduction}

Stroke is a clinical syndrome characterized by the rapid onset of focal neurological signs, lasting more than 24 hours or leading to death, with a presumed vascular cause, either due to infarction or haemorrhage. The most striking finding of stroke is hemiplegia, a clinical condition including motor deficits, sensory disorders, and various neurological manifestations mainly in contralateral half of the body. Consequently, manipulative behaviors, based on upper extremity function and used during daily routines and professional life, are often impaired in brain-damaged patients $[1,2]$. Despite advances in medical care and rehabilitation, the recovery of hand function following stroke is often limited [3]. The primary goal of stroke rehabilitation is to make patients independent with their daily living activities and this achievement is mainly possible with appropriate functioning of the hand and upper extremity.

Hand dominance refers to a hand preference when performing a task. Hand dominance is an important factor in the performance of motor skills, because the dominant hand is used for many daily and recreational activities. The speed, precision, and coordination of the dominant hand are superior to those of the non- dominant hand, which may stem from motor programs and skills developed through extensive practice and experience associated with the use of dominant hand [4].

Stroke patients experience serious disability when the effected hand is the dominant side. They have to depend on 
the nondominant hand for their activities of daily living. However we assumed that this disadvantage might end up in better results with the more effort of the patient for recovery of the dominant hand.

In this study, we aimed to investigate the controversy about the effect of hand dominance on both functional loss and regain after stroke.

\section{Methods}

18 patients with either right or left hemiplegia participated in the study. Patients were recruited from the Mersin University Hospital Neurology Clinics. Aphasia, orientation problems, dementia, neglect, complex regional pain syndrome, previous upper extremity tendon and nerve injuries, fractures and comorbid inflammatory/ neurological systemic diseases were accepted as exclusion criteria for the study. Patients were informed about the whole procedure and written and oral consent is obtained as well as the acceptance of the local ethics comitte (Mersin University). Patients were grouped as dominant side and nondominant side hemiplegic (groups D and ND). Information on hand dominance was obtained by asking the individual which hand they preferred to use for writing and throwing a ball prior to the stroke. This information was then coded into 0 (dominant hand affected) or 1 (non-dominant hand affected).

All patients in the two groups were evaluated in the $1^{\text {st }}$ week, $1^{\text {st }}$ month and $3^{\text {rd }}$ month after stroke. Motricity index, Modified Ashworth Scale (MAS) and Functional Independence Measure (FIM) were recorded for every patient in each visit. Brunnstrom motor recovery stages for both upper extremity and hand has been recorded. Dexterity was observed with Nine Hole Peg Test (NHPT), hand grip strength was determined with Jamar dynamometer and pinchmeter. Measurement and evaluation procedures have been applied by the same physician at the same room approximately at the same time of the day.

Modified Ashworth Scale (MAS):

The Ashworth Scale measures spasticity according to the following scale; 0: no increase in muscle tone, 1: slight increase in muscle tone, manifested by a catch and release or by minimal resistance at the end of the range of motion when the affected part is moved in flexion or extension, 2 : marked increase in muscle tone, manifested by a catch in the middle range and resistance throughout the remaining range of motion, but affected part easily moved, 3: considerable increase in muscle tone, passive movement difficult, and $4=$ affected part rigid in flexion or extension [5]. The Modified Ashworth scale includes a score of 1+ (slight increase in tone with minimal resistance through less than half range) which is distinctive from a score of 1 where the resistance is felt only at end range [5]. The standardized procedure for the study was as follows: each subject was put in a resting position for 5 minutes. Each test movement was performed over a duration of about 1 second (by counting "one thousand one"), as described by Bohannon and Smith $[6,7,8]$.

\section{Brunnstrom Recovery Stages (BRS):}

BRS is a well-known measure for modeling the recovery process, following stroke-induced hemiplegia used for stroke patients. It has is a six-stage-evaluation tool and has three different parts concerning the upper extremity, hand and the lower extremity. The Brunnstrom approach is focused on the progressive development of the motion synergic pattern during the rehabilitation process and divides the motor recovery into six stages from the period of complete flaccidity to the disappearance of spasticity when the patient is able to perform near-normal to normal movement. The simplicity makes the Brunnstrom classification one of the most favorable measures as a repetitive follow-up test during rehabilitation program $[9,10]$.

\section{Brunnstrom Staging for Hand}

\section{Stage 1: Flaccidity}

Stage 2: Little or no active finger flexion

Stage 3: Mass grasp; use of hook grasp but no release; no voluntary finger extension; possible reflex extension of digits

Stage 4: Lateral prehension, release by thumb movement; semi-voluntary finger extension of digits, variable range

Stage 5: Palmar prehension; possibly cylindrical and spherical grasp, awkwardly performed and with a limited functional use; voluntary mass extension of the digits, variable range

Stage 6: All prehensile types under control; skills improving; full range voluntary extension of the digits; individual finger movements present, less accurate than on the opposite side.

Nine Hole Peg Test (NHPT): NHPT is a time test for quantitative assessment of manual dexterity. In the last few years, the NHPT has been one of the most frequently used measures of upper extremity function in various conditions like carpal tunnel syndrome, tendon injuiries, amputations as well as MS, stroke, head injury and polyneuropathies. As a small instrument, it is easy to carry and use where suitable, even in bed. The NHPT provides a brief, standardized approach to assessment and can be administered by a wide variety of trained examiners. Also this test has high interrater reliability and good test-retest reliability [11].

The NHPT consists of 9 pegs and a peg board with 9 holes spaced $2.54 \mathrm{~cm}$ apart. The patient picks up the nine pegs one at a time as quickly as possible, puts them in the nine holes, and, once they are in the holes, removes them again as quickly as possible one at a time, replacing them into the container $[12,13]$. The total time to complete the task is recorded.

Performance on the 9-Hole Peg Test may be sensitive to practice effects, that is, patients often display poorer performance when first tested due to lack of familiarity with the task. It is recommended that three or four administrations be given prior to a baseline assessment if accurate (rather than comparative) assessments of change over time are needed.

Normative data for adults are available for NHPT [11].

Motricity Index:

Motricity Index is used to asses motor impairment and 
recovery in stroke patients [14]. With the patient seated, power and range of active movement are rated for shoulder abduction, elbow flexion, and pinch between the thumb and index finger. These were each scored (0-33) according to the instructions of Collin and Wade [15]. The total upper extremity score is involved adding one to the sum of the three actions (maximum possible score $=100$ ) [16].

Grip strength:

Grip strength was assessed using a hand held dynamometer (Jamar (C). The participants were told to keep the shoulder adducted, the elbow flexed at 90 degrees, and the forearm and wrist in the neutral position without resting the arm on the table or chair and to place his or her fingers on the second position of the handle of the dynamometer. The display was then set to zero, thus discounting any resting pressure exerted by the patient due to involuntary flexion of the fingers. The patients were told to squeeze as hard as possible and then release. Verbal encouragement was provided whenever necessary [17]. This procedure was repeated three times and mean score of the measurements was recorded

\section{Pinch strength:}

3 types of pinch can be measured: lateral (key) pinch, pulp (tip) pinch and palmar pinch. We used pulp pinch strength assessed with the pinchmeter. While measuring the pulp pinch, the pinchmeter is held between the tip of the thumb and the tip of the index finger [18]. Just like grip strength we also completed three measurements and the mean value was recorded for each position.

Functional Independence Measure:

Most recently, the functional independence measure (FIM) has become the predominant tool for measuring multiple disabilities in studies. The FIM, which is historically derived from the Barthel Index, is primarily an ordinal scale with some interval characteristics. FIM evaluates 18 items under a 7-level scale of independent performance in self- care, sphincter control, transfers, locomotion, communication, and social cognition. Thirteen of the items compose the motor subscale (FIM motor), and the remaining 5 items make up the cognitive subscale (FIM cognitive) [19]. In previous literature, the FIM motor and FIM cognitive have been found to define separate constructs, for this investigation, we focused on the FIM motor subscale only [20]. Each of these activities is evaluated and receives a score ranging from one (total dependence) to seven (complete independence) and the total score ranges from 13 to 91 for motor FIM.

The FIM has been documented to be a valid and reliable measure of disability and a useful screening tool [21,22].

\section{Statistics}

For statistical analysis SPSS Statistical Package for the Social Sciences) for Windows 16 has been used. Homogenity of the variables were tested with Shapiro Wilk test. For the scores, variables has been summarized as [min.-max.]. We compared the group data for variables with Mann Whitney U and Friedman tests. Spearman's correlation coefficient was used to display relationships between measurements. For all tests, $\mathrm{p}$ values $<0.05$ is accepted as statistically significant.

\section{Results}

18 hemiplegic patients were recruited from the Mersin University Hospital. All patients were hospitalized for stroke at the Neurology Department. All were ischemic stroke, 15 were MCA (middle cerebral artery) and 3 were ACA (anterior cerebral artery) infarcts.

After the exclusion criteria evaluation, patients were examined and enrolled in the study. The evaluation procedure has been repeated three times during the study; at the $1^{\text {st }}$ week (visit 1), $1^{\text {st }}$ month (visit 2) and $3^{\text {rd }}$ monthS (visit 3) after stroke.

Five of all patients were female and 13 were male, with an age range between 45 and 81 years. All patients were right handed except one.

7 patients were grouped as the dominant side stroke group (D) as they had hemiplegia on the dominant hand side. They were all right handed. There was no left handed dominant side stroke patients. 1 patient was female, others were male with ages between 45 and 80 (mean $59.374 \pm 13.64$ years)

The nondominant side stroke group (ND) consisted of 11 patients. 10 were right handed left hemiplegic, and one was vice versa. This group consisted of 4 female and 7 male patients. The average age for this group was $63.72 \pm 12.6$

The D and ND groups were found to be similar for age, gender and etiologic- demographic factors. These variables are given in Table 1.

Baseline evaluation for Brunnstrom and MAS were similar with no significant diffences between groups for the first visit. This similarity remained throughout the study $(p>0,05)$. The correlation analysis between MAS and Brunnstrom levels revealed a positive correlation for both groups and for each visit.

Fine and gross grip evaluation recorded as pinch strength scores and Jamar dynamometry grip results displayed no statistically significant difference between groups for all three visits recorded. ( $p>0.05$ for all). The 2 grip type results showed a good correlation with each other for all patients.

NHPT was used to assess fine manual dexterity and the difference between NHPT scores at the initial examination were not statistically significant. However at the third visit, they revealed a significant difference in the final analysis $(\mathrm{p}<0,05)$.

FIM and Motricity Index did not show a significant difference between groups throughout the whole follow up period and remained similar $(\mathrm{p}>0,05)$.

After comparing patients according to hand dominance groups, we also investigated the correlations between outcome measures. These correlation analysis displayed a positive correlation between hand grip strength and FIM values $(r=0.645, p<0.05)$ for both groups. A similar correlation was present between pinch grip strength and NHPT scores too $(\mathrm{r}=0.628, \mathrm{p}<0.05)$. 


\section{Discussion}

In stroke, loss of arm-hand function and consequently, loss of arm-hand performance leads to major problems in the everyday life of these patients. It limits the execution of activities of daily living, which results in greater dependency, restricted social participation, and a decreased quality of life. Four years after stroke, $67 \%$ of the patients experience the non-use or disuse of the affected arm as a major problem, whereas only $6 \%$ of the patients is fully satisfied with their arm-hand function [23].

Hand dominance refers to a hand preference when performing a task and an important factor in the performance of motor skills. The speed, precision, and coordination of the dominant hand are superior to those of the non-dominant hand, which may stem from motor programs and skills developed through extensive practice and experience associated with the use of dominant hand. Also muscle fatigue has been found to be greater in the non-dominant hand. Walsh et al. found that a large portion (up to 35\%) of individuals with hand injuries had to change handedness post injury and reported functional loss [8].

Common upper extremity (UE) impairments after stroke include 1- paresis, 2- loss of fractionated movement, 3abnormal muscle tone and/or 4- changes in somatosensation. Clinically, paresis appears as weakness and results in slower, less accurate, and less efficient movements compared with those in neurologically intact individuals [24]. Muscle tone alterations may effect the movement negatively too either as spasticity or flaccidity. Fractionation of movement is the ability to voluntarily move one segment independently of other segment and is essential for skilled UE motor control. The stroke-induced damage to fractionate movement can limit UE function [24]. If stroke damages the ascending somatosensory pathways and/or cortical areas, then individuals will have a reduction or loss of somatosensation and the nervous system will have less ability to monitor and correct the movements [24].

Although each of the impairments listed above can occur in isolation, more often they exist in combinations. Therefore any valid evaluation process must determine the presence and severity of these impairment and how the impairments are contributing to the loss of movement and function [24].

Numerous measures are readily available to clinicians for the evaluation of UE function after stroke and many of these measures have been thoroughly evaluated for reliability and validity at multiple time points after stroke.

These measures can be generally divided into two categories: 1) performance measures, where the clinician rates or times a series of UE actions that are performed by the patient or 2) self-report measures, where the clinician asks a series of questions about UE actions that are answered verbally by the patient. On the other hand, actual performance can be measured only by direct and objective assessment in the real-life situation [23] To determine the capacity of the affected arm-hand, unimanual tasks are useful because these tasks force the use of the affected arm-hand.
However, in daily life, many tasks are bimanual requiring both hands to perform the tasks. More over in daily life, the affected arm-hand is rarely used for unimanual tasks. Therefore, if assessment in daily life is aimed for, bimanual items should be included [23].

Once a measure is selected, the same measure should be administered at evaluation, interim and discharge evaluations to document progress or lack thereof. We selected grip strength, Motricity index, NHPT and FIM for this study to evaluate different aspects-dimensions of disability, in addition to the standard follow up procedures with Brunnstrom and Ashworth Scales.

After stroke most motor and functional recovery will occur within the first three months. There are three indicators of future outcomes that are useful to look for when trying to determine prognosis in individuals. First, the accompanying nonmotor impairments (e.g., somatosensory loss or visual field loss) are considered to be no good. Second, early, rapid improvements in motor impairments are indicators that a person is more likely to reach higher levels of independence. And third, the absence of measurable grip strength or shoulder flexion at three to four weeks after stroke attack is a strong indicator that the affected upper limb will be nonfunctional.Evaluation in a few week after stroke can predict later UE function A few days after stroke, measurements indicate the initial severity of paresis, but not the rate of change. By three weeks after stroke, measurements reflect both [24]. We made our study plan so as to evaluate the patients both about rapid improvement and the rate of change with three visits at first week, first month and third month after stroke

Grip and pinch strength are common hand function tests recommended for stroke patients [25, 26]. It shows good sensitivity to change, detecting early improvement. Sunderland et al. assessed 4 upper extremity tests (Frenchay Arm Test, Nine Hole Peg Test, Motricity Index, and Motor Club Assessment) and grip strength measures. Of particular interest were the results for strength of the grip which showed that it was the best of these measures for detecting early recovery and was useful in predicting the final outcome [27]. The absence of measurable grip by one month after stroke, indicates that there will be poor functional outcome. All our patients were able to grip the dynamometer even at first week. Unfortunately measuring the grip strength of stroke patients has been rejected actively within orthodox physiotherapy. This rejection has been motivated by two concerns. First, that measuring strength alone ignores the role of impaired coordination of muscle groups in producing deficient motor performance. We in our study overcame this disadvantage by using a variety of tests. Second because an increase in finger flexion is part of the spastic pattern which typically evolves after stroke, increased grip might indicate this spasticity rather than any improvement in muscle control and it would be difficult to distinguish voluntary grasp from spastic flexion of the fingers. We admit that we cannot exclude the possibility that spasticity might contribute to grip strength in some cases. However, as all patients in our study 
patients had similar MAS results and Brunnstrom stages, we suggest that if there is such a contribution, it can be ignored, and does not invalidate this measure as an index of voluntary function.

As we did for our results, using mean score is better than using highest score to represent the muscle strength in clinical research $[25,28]$. We found that all three trials may differ in strength tests due to either tiredness or adaptation. Therefore, it is more effective and reliable to choose the mean score of multiple trials.

The Nine-Hole Peg Test is a brief measure used to evaluate small muscles and quantify hand dexterity. In the test, performance is quantified as the time taken to place and then remove the pegs, one at a time and suitability for active rehabilitation is defined as the ability to complete the peg test with the unaffected hand within a generous time limit of 30 seconds. This excludes patients who are very drowsy or who have very severe cognitive deficit [27]. So NHPT is also an indicator for rehabilitation potential besides being an outcome measure

In the literature the relationship between grip strength and the NHPT has been reported to be r: 0.71 and r. 0.79 at 1 and 6 months respectively. However in our study NHPT scores were well correlated with pinch grip strength, not grip This seemed logical to us since NHPT involves the coordination of several fingers within a hand and/or coordinated action of fingers, not just grasping [29]. So pinch strength measure can be an indicator of fine motor function and a way much easier method.

Given the fact that the NHPT requires greater finger motor control, like grip and pinch strength, it is not surprising that performance can be susceptible to muscle tone variations [26]. But as we stated before we believe our measurements were unaffected from these changes.

The Motricity Index, involves grading strength on the basis of a patient's ability to activate a muscle group, to move a limb segment through a range of motion, and to resist the force of an examiner and is accepted as another accurate prediction of outcome. Bard and Hirschberg found that patients who eventually gained full range of movement had visible movement at the shoulder, elbow or hand within the first month. The validity of the Motricity Index for the upper extremity is supported by the high degree correlation with both grip strength and a measure of upper extremity function in previous studies [30].

The present results provide support for the use of the Motricity Index for characterizing the strength of the paretic upper extremity following stroke [31]. On the other hand, this test is probably useless in planning physiotherapy treatment as it gives no information on quality of performance. Besides it is probably not sensitive to minor changes.

This again confirms the argument that ideal assessment of the arm should not be based on a single measure, but should include a selection of tests of sensation, co-ordination and dexterity. We chose dexterity, strength and ADL to quantify stroke related disability. We suggest that when selecting upper extremity function tests for individual patients, the circumstances can dictate the test choice, instead of any one test being considered the "gold standard". In other words, the choice of which test to use could be made based on the individual patient and what is available in the therapist's practice setting [32].

Hand dominance has been cited as an important factor in the performance of motor skills. Studies report between 45$50 \%$ of individuals sustain a left hemisphere lesion and therefore right side paresis Since up to $80 \%$ of people are right hand dominant, a significant proportion of individuals who experience a stroke will have their dominant hand affected.

Not much studies have examined the impact having the dominant versus the non-dominant hand affected post stroke has on impairment, activity, and participation. However, given the findings from studies on both the neurological and peripheral changes in arm function post stroke, we hypothesized that individuals with their dominant hand affected by the stroke would experience more impairment, and worse performance in ADL compared to those with their non-dominant hand affected especially at early stages of recovery. But on the other hand we assumed that this disadvantage may lead to greater effort to regain hand function in the rehabilitation process unless the degree of paralysis is nor severe enough to preclude possible functional movement.

If the dominant hand has been affected by the stroke, individuals may be more motivated to use their dominant hand during recovery since they are not used to using their non-dominant hand for daily tasks. In contrast, if the nondominant hand is affected, individuals may have little motivation to use this hand in daily tasks making it difficult to promote the use of the non-dominant hand in therapy

Besides the propensity to use the dominant hand may lead to a better pre-stroke neuromuscular condition of the dominant hand (e.g., stronger muscles, more efficient motor unit recruitment) compared to the non-dominant hand. Provins concluded that there is a preference to utilize the dominant arm more often during daily activities and this is reflected by better arm pointing accuracy, movement speed, and precision when using the dominant hand in healthy adults [33].

Observations suggest less impairment and/or better recovery in the index and middle fingers of the affected hand. Less impairment in these two fingers may be causally related to their more frequent use during daily activities [34]. This theory may apply to the whole hand, regarding frequent use of the dominant hand. Therefore, if the dominant hand is affected by the stroke it may demonstrate less impairment immediately following the stroke due to its protective effect. One study directly compared the paretic limbs of patients with left or right hemisphere stroke and found that basic motor functioning (eg, tone, grip strength) was better in the paretic limb when it was the preferred limb, which was attributed to the preferred limb's better neuromuscular condition at the first month [34]. Muscle fatigue has been 
found to be greater in the non-dominant hand.

Harris and Eng investigated whether the impairment in patients with chronic stroke was affected by the involvement of dominant hand. They found less impairment when the dominant hand was involved, compared to the involvement of the non-dominant hand. Thus, in their study population although dominance had a positive effect on the impairment, it did not translate into better arm performance in ADL [8].

Rinehart et al measured arm use quantitatively in a righthanded healthy control group (HC) and right-handed patients with stroke with right or left hemiplegia as they performed activities of daily living. They predicted: the right nonparetic limb in patients with left hemiplegia would be used more frequently than the left nonparetic limb in patients with right hemiplegia. and the right hemiplegia group would use both arms together more than the left hemiplegic group [34].

Overall, it is acknowledged that both handedness groups have distinctive activation patterns with left-handers showing less hemispheric asymmetries than right-handers when performing intricate motor tasks [35,36].

On the contrary even more fundamental is the recent recognition by several investigators that the nonparetic upper extremity's performance in poststroke patients is both slower and less consistent than that of healthy subjects. Diminished performance of the nonparetic limb may be attributed to a variety of factors, including microdamage in the "unaffected hemisphere" that current imaging technologies are unable to detect, altered interhemispheric metabolism or unknown causes. We did not investigate or test the unaffected hand here.

In a study Nam et al investigated whether the paralysis of dominant hand affected QOL in patients with subacute stroke. They found no significant differences in QOL, ADL, or depression between patients with paralysis of the dominant hand and those with paralysis of the non- dominant hand [4]. We too couldn't display FIM differences between dominant and nondominant side hemiplegic groups in a 3 month period. Having the dominant hand affected did not impact functional measures. Thus those with stronger grip strength due to dominance showed no greater functional independence over those with the non-dominant hand affected at the subacute stage. Our results reflect the events in the acute- subacute stage too. Final functional state may be different involving compensation and adaptation mechanisms. Also rehabilitation process affects the outcome even in similar cases.

Individuals may benefit from grip strength and gripping activities (e.g. grasp/release, turning, pushing/pulling) during rehabilitation to minimize joint stiffness, increase/maintain range of motion, and help prevent shoulder-hand syndrome. So the presence of grip ability is not just an indicator or test result but a valuable component of rehabilitation process.

\section{Conclusions}

In the light of these data, we concluded that there was no significant difference in functional improvement between dominant and non-dominant side hemiplegic groups during the first three months after stroke. Grip and pinch strength measurements are easy to apply and reliable to follow up hand functions. They might be an indicator when using multiple measures are not possible due to time or patient or clinical setting problems. We admit the small size of the study total group and consequently left handed patient group is a pitfall of our study. More studies with longer follow ups and larger patient groups are needed to clarify the effect of hand dominance on long term functional status of the patient

Table 1. Demographic information about groups.

\begin{tabular}{lll}
\hline Sex & Male & 13 \\
& Female & 5 \\
Dominant hand & Right & 17 \\
& Left & 1 \\
Hemiplegic side & Right & 8 \\
& Left & 10 \\
\hline
\end{tabular}

\section{References}

[1] B. S., "Motor testing procedures in hemiplegia: based on sequential recovery stages.," Phys Ther, no. 46, pp. 357-75, 1966.

[2] M. Wiesendanger and D. J. Serrien, "Neurological problems affecting hand dexterity.," Brain Res. Brain Res. Rev., vol. 36, no. 2-3, pp. 161-8, Oct. 2001.

[3] M. N. McDonnell, S. L. Hillier, M. C. Ridding, and T. S. Miles, "Impairments in precision grip correlate with functional measures in adult hemiplegia.," Clin. Neurophysiol., vol. 117, no. 7, pp. 1474-80, Jul. 2006.

[4] H. U. Nam, J. S. Huh, J. N. Yoo, and J. M. Hwang, "Effect of Dominant Hand Paralysis on Quality of Life in Patients With Subacute Stroke," vol. 38, no. 4, pp. 450-457, 2014.

[5] T. Kaya, A. Goksel Karatepe, R. Gunaydin, A. Koc, and U. Altundal Ercan, "Inter-rater reliability of the Modified Ashworth Scale and modified Modified Ashworth Scale in assessing poststroke elbow flexor spasticity," Int. J. Rehabil. Res., vol. 34, no. 1, pp. 59-64, Mar. 2011.

[6] R. W. Bohannon, a. W. Andrews, and M. B. Smith, "Rehabilitation goals of patients with hemiplegia," Int. J. Rehabil. Res., vol. 11, no. 2, pp. 181-184, Jun. 1988.

[7] M. Blackburn, P. van Vliet, and S. P. Mockett, "Reliability of measurements obtained with the modified Ashworth scale in the lower extremities of people with stroke.," Phys. Ther, vol. 82, no. 1, pp. 25-34, 2002.

[8] J. J. E. Jocelyn E Harris, "Individuals with the dominant hand affected following stroke demonstrate less impairment than those with the non-dominant hand affected," Neurorehabil. Neural Repair, vol. 20, no. 3, pp. 380-389, 2006.

[9] B. Chung, "Effectiveness of Extracorporeal Shock Wave Therapy in the Treatment of Previously Untreated Lateral Epicondylitis: A Randomized Controlled Trial,' Am. J. Sports Med., vol. 32, no. 7, pp. 1660-1667, Oct. 2004.

[10] Z. Zhang, Q. Fang, and X. Gu, "Objective Assessment of Upper Limb Mobility for Post-stroke Rehabilitation," IEEE Trans. Biomed. Eng., vol. 9294, no. c, pp. 1-1, 2015. 
[11] V. Mathiowetz, K. Weber, N. Kashman, and G. Volland, "Adult norms for the Nine Hole Peg Test of finger dexterity.," Occup. Ther. J. Res., vol. 5, no. 1, pp. 24-38, 1985.

[12] G. Information and P. Instructions, "Nine Hole Peg Test Instructions General Information :," pp. 1-3, 1985.

[13] K. O. Grice, K. a. Vogel, V. Le, A. Mitchell, S. Muniz, and M. A. Vollmer, "Adult norms for a commercially available nine hole peg test for finger dexterity," Am. J. Occup. Ther, vol. 57, no. 5, pp. 570-573, 2003.

[14] I. Safaz, B. Yilmaz, E. Yaşar, and R. Alaca, "Brunnstrom recovery stage and motricity index for the evaluation of upper extremity in stroke: analysis for correlation and responsiveness.," Int. J. Rehabil. Res., vol. 32, no. 3, pp. 228 31, Sep. 2009.

[15] F. M. Collen, D. T. Wade, and C. M. Bradshaw, "Mobility after stroke: reliability of measures of impairment and disability.," Int. Disabil. Stud., vol. 12, no. 1, pp. 6-9.

[16] C. Collin and D. Wade, "Assessing motor impairment after stroke: a pilot reliability study.," J. Neurol. Neurosurg. Psychiatry, vol. 53, no. 7, pp. 576-9, Jul. 1990.

[17] R. W. Bohannon, J. Bear-Lehman, J. Desrosiers, N. MassyWestropp, and V. Mathiowetz, "Average grip strength: a metaanalysis of data obtained with a Jamar dynamometer from individuals 75 years or more of age.," J. Geriatr. Phys. Ther., vol. 30, no. 1, pp. 28-30, Jan. 2007.

[18] V. Mathiowetz, C. Rennells, and L. Donahoe, "Effect of elbow position on grip and key pinch strength.," J. Hand Surg. Am., vol. 10, no. 5, pp. 694-697, 1985.

[19] J. Sengler, E. Hartmann, P. Buisson, C. Pierrejean, and D. Bourderont, "Is functional independence measure (FIM) predictive for stroke rehabilitation?," Ann. Readapt. Med. Phys., vol. 39, pp. 553-559, 1996.

[20] C. Glenny, P. Stolee, M. Thompson, J. Husted, and K. Berg, "Underestimating physical function gains: comparing FIM motor subscale and interRAI post acute care activities of daily living scale.," Arch. Phys. Med. Rehabil., vol. 93, no. 6, pp. 1000-8, 2012.

[21] H. Arai, Y. Ouchi, M. Yokode, H. Ito, H. Uematsu, F. Eto, S. Oshima, K. Ota, Y. Saito, H. Sasaki, K. Tsubota, H. Fukuyama, Y. Honda, A. Iguchi, K. Toba, T. Hosoi, and T. Kita, "Toward the realization of a better aged society: messages from gerontology and geriatrics.," Geriatr. Gerontol. Int., vol. 12, no. 1, pp. 16-22, Jan. 2012.

[22] M. Inouye, K. Kishi, Y. Ikeda, M. Takada, J. Katoh, M. Iwahashi, M. Hayakawa, K. Ishihara, S. Sawamura, and T. Kazumi, "Prediction of functional outcome after stroke rehabilitation.," Am. J. Phys. Med. Rehabil., vol. 79, no. 6, pp. 513-8, 2000 .

[23] R. J. M. Lemmens, A. a a Timmermans, Y. J. M. JanssenPotten, R. J. E. M. Smeets, and H. a M. Seelen, "Valid and reliable instruments for arm-hand assessment at ICF activity level in persons with hemiplegia: a systematic review.," $B M C$ Neurol., vol. 12, no. 1, p. 21, Jan. 2012.
[24] C. E. Lang, M. D. Bland, R. R. Bailey, S. Y. Schaefer, and R. L. Birkenmeier, "Assessment of upper extremity impairment, function, and activity after stroke: foundations for clinical decision making.," J. Hand Ther, vol. 26, no. 2, p. 104-14; quiz 115, 2013.

[25] S.-L. Huang, C.-L. Hsieh, J.-H. Lin, and H.-M. Chen, "Optimal scoring methods of hand-strength tests in patients with stroke.," Int. J. Rehabil. Res., vol. 34, no. 2, pp. 178-80, Jun. 2011.

[26] H.-M. Chen, C. C. Chen, I.-P. Hsueh, S.-L. Huang, and C.-L. Hsieh, "Test-retest reproducibility and smallest real difference of 5 hand function tests in patients with stroke.," Neurorehabil. Neural Repair, vol. 23, no. 5, pp. 435-40, Jun. 2009.

[27] A. Sunderland, D. Tinson, L. Bradley, and R. L. Hewer, "Arm function after stroke. An evaluation of grip strength as a measure of recovery and a prognostic indicator.," J. Neurol. Neurosurg. Psychiatry, vol. 52, no. 11, pp. 1267-72, Nov. 1989.

[28] E. Svensson, K. Waling, and C. Häger-Ross, "Grip strength in children: test-retest reliability using Grippit.," Acta Paediatr., vol. 97, no. 9, pp. 1226-31, Sep. 2008.

[29] S. Li, M. L. Latash, G. H. Yue, V. Siemionow, and V. Sahgal, "The effects of stroke and age on finger interaction in multifinger force production tasks," Clin. Neurophysiol., vol. 114, no. 9, pp. 1646-1655, Sep. 2003.

[30] A. Sunderland, D. Tinson, L. Bradley, and R. L. Hewer, "Arm function after stroke. An evaluation of grip strength as a measure of recovery and a prognostic indicator.," J. Neurol. Neurosurg. Psychiatry, vol. 52, no. 11, pp. 1267-1272, Nov. 1989.

[31] R. W Bohannon, "Motricity Index Scores are Valid Indicators of Paretic Upper Extremity Strength Following Stroke.," J. Phys. Ther. Sci., vol. 11, no. 2, pp. 59-61, 1999.

[32] J. A. Beebe and C. E. Lang, "Relationships and responsiveness of six upper extremity function tests during the first six months of recovery after stroke.," J. Neurol. Phys. Ther., vol. 33, no. 2, pp. 96-103, Jun. 2009.

[33] K. A. Provins and J. Magliaro, "The measurement of handedness by preference and performance tests.," Brain Cogn., vol. 22, no. 2, pp. 171-181, 1993.

[34] J. K. Rinehart, R. D. Singleton, J. C. Adair, J. R. Sadek, and K. Y. Haaland, "Arm use after left or right hemiparesis is influenced by hand preference.," Stroke., vol. 40, no. 2, pp. 545-50, Feb. 2009.

[35] Y. Laufer, L. Gattenio, E. Parnas, D. Sinai, Y. Sorek, and R. Dickstein, "Time-related changes in motor performance of the upper extremity ipsilateral to the side of the lesion in stroke survivors.," Neurorehabil. Neural Repair, vol. 15, no. 3, pp. $167-172,2001$.

[36] D. J. Serrien and M. M. Sovijärvi-Spapé, "Cognitive control of response inhibition and switching: hemispheric lateralization and hand preference.," Brain Cogn., vol. 82, no. 3, pp. 283-90, Aug. 2013. 